\title{
Actualización de los programas de detección precoz de la sordera infantil: recomendaciones CODEPEH 2018 (Nivel 1: Detección)
}

\author{
Update in early detection \\ of pediatric hearing loss: \\ 2018 CODEPEH recommendations \\ (Level 1: Screening)
}

\section{Palabras clave}

Hipoacusia, cribado neonatal hipoacusia, pérdidas en el seguimiento, infección congénita citomegalovirus, diagnóstico etiológico de la hipoacusia infantil.

\section{Keywords}

Hearing loss, newborn hearing screening, loss to follow-up, congenital cytomegalovirus infection, etiological diagnosis of pediatric hearing loss.

1. Introducción

Han pasado ocho años desde que la CODEPEH publicó el Documento de Recomendaciones en el que se destacaba el importante papel que tienen la detección precoz y la intervención temprana en el aprendizaje del lenguaje oral por parte de los niños con sordera y así disponer de una importante herramienta que permita la inclusión plena en la sociedad (Trinidad et al., 2010: 69-77). Las actualizaciones de los protocolos de cribado neonatal de la hipoacusia descritos en este documento se consideraron de referencia y fueron adoptadas en muchos programas de cribado, asumiendo como hechos constatados que:

1. La hipoacusia congénita bilateral permanente tiene una incidencia de uno a cinco niños por 1000 nacimientos. Esta cifra es superior a cualquiera de las enfermedades metabólicas que se criban mediante la "prueba del talón".

\footnotetext{
1. El presente Documento de Recomendaciones CODEPEH 2018 ha sido elaborado en el marco del proyecto sobre la Actualización de los Programas de Detección Precoz de la Sordera Infantil, que ha sido desarrollado por la Confederación Española de Familias de Personas Sordas-FIAPAS, en colaboración con la Comisión para la Detección Precoz de la Sordera Infantil-CODEPEH, con la coorganización del Real Patronato sobre Discapacidad.
}

Faustino Núñez Batalla <fnunezb@uniovi.es>

Presidente de la CODEPEH. Servicio ORL, Hospital Universitario Central de Asturias-Oviedo. Sociedad Española de Otorrinolaringología. España

\section{Carmen Jáudenes \\ Casaubón}

<direccion@fiapas.es>

Vocal de la CODEPEH. Directora de la Confederación Española de Familias de Personas Sordas (FIAPAS). España

\section{José Miguel Sequí Canet}

Vocal de la CODEPEH. Jefe de Servicio de Pediatría, Hospital de Gandía-Valencia. Asociación Española de Pediatría. España

\section{Ana Vivanco Allende}

Vocal de la CODEPEH. Servicio de Pediatría, Hospital Universitario Central de Asturias-Oviedo. Asociación Española de Pediatría. España

\section{José Zubicaray Ugarteche}

Vocal de la CODEPEH. Servicio ORL Infantil, Complejo Hospitalario de Navarra-Pamplona. Sociedad Española de Otorrinolaringología. España

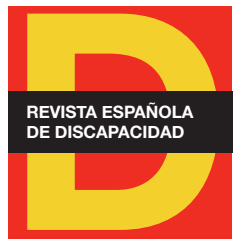

Para citar:

Núñez, F. et al. (2019): “Actualización de los programas de detección precoz de la sordera infantil: recomendaciones CODEPEH 2018 (Nivel 1: Detección)". Revista Española de Discapacidad, 7 (I): 201-220.

Doi: <https://doi.org/10.5569/23405104.07.01.10> 
2. La sordera, sin el apropiado tratamiento temprano, tiene graves consecuencias para el niño y su familia, dado que es necesario contar con una correcta audición en los períodos críticos de la infancia para lograr su óptimo desarrollo.

3. Está demostrado que las tecnologías actuales (otoemisiones acústicas y potenciales evocados de tronco cerebral automatizados) son lo suficientemente precisas, fiables, objetivas y coste-efectivas en el papel que se les asigna para la detección precoz de la hipoacusia (Nikolopoulos, 2015: 635-637).

Existen diversos estudios que confirman el hecho de que el cribado universal acorta los plazos tanto de diagnóstico como de intervención en casos de hipoacusia (Wake et al., 2016: 1-10; Korver et al., 2010: 17011708; Kennedy et al., 2005: 660-662; Kennedy, 1999: 73-75). En comparación con la media de dos años de edad al diagnóstico en los casos no sometidos a cribado, se ha demostrado que con el cribado neonatal se puede confirmar el diagnóstico de hipoacusia antes de los seis meses de vida (Wood et al., 2015: 353-358). Las consecuencias del adelanto en el diagnóstico y el tratamiento de la sordera en lo relativo a la adquisición del lenguaje y la comprensión lectora está demostrado que son positivas, incluso existen evidencias de su utilidad más allá de la edad escolar (Pimperton et al., 2017: 598-610; Bruijnzeel et al., 2016: 113-126; Pimperton et al., 2016: 9-15; Ching et al., 2013: 535-552; Kennedy et al., 2006: 2131-2141).

\subsection{Puntos de actualización}

Aunque los programas de detección precoz de la hipoacusia congénita hayan superado los errores conceptuales esgrimidos para oponerse a su implantación y se han extendido de forma exitosa, sobre todo en los países desarrollados, actualmente aún se pueden identificar numerosas dificultades y debilidades que los amenazan. El análisis de estos problemas permite identificar una serie de puntos sobre los cuales actuar con el fin de mejorarlos.

Según se han ido implantando los programas, la edad de los niños al diagnóstico de la hipoacusia ha ido descendiendo desde los dos años de edad hasta los pocos meses de vida. Sin embargo, no es raro encontrar actualmente niños con una hipoacusia profunda, que incluso precisó de implante coclear, que habían pasado con éxito el cribado neonatal.

Estos niños con hipoacusia de desarrollo tardío (en algunos casos relacionada con la infección congénita por citomegalovirus o con alteraciones genéticas) se encuentran en una peor situación que aquellos que no pasaron el cribado, dado que el diagnóstico suele retrasarse por la falsa creencia de que superarlo asegura contar con una audición normal de forma permanente.

Otra importante debilidad de los programas es el número de niños que no pasan el cribado y que no completan el proceso de diagnóstico, por lo que no reciben un tratamiento temprano. El porcentaje de niños sin superar el cribado que se pierden en el seguimiento llega a ser alarmante (se encuentran cifras entre el 20 y el $50 \%$ ), hecho que amenaza los objetivos de los programas. El análisis de este problema ha demostrado que este fenómeno está relacionado en buena medida con la actitud parental ante el proceso y con factores socioeconómicos de la familia sobre los que se puede actuar (Bush et al., 2017: S1-13). No obstante, pueden influir otros factores.

Aunque los programas de cribado, diagnóstico y tratamiento precoz de la sordera infantil tienen ya un largo recorrido, es importante recordar y actualizar los principios básicos que pueden asegurar su éxito mediante 
la formación continuada de los profesionales que integran el equipo interdisciplinar, piedra angular de los mismos. Por todo ello, la CODEPEH considera necesario formular unas nuevas recomendaciones que pueden contribuir a la actualización de los programas de cribado auditivo con diversas evidencias aparecidas en la última década. En estas recomendaciones se abordan los progresos vinculados con el primer nivel de la aplicación de los programas: la detección. Se trata de los relativos a los protocolos y la tecnología de cribado, el impacto que tienen los conocimientos actuales sobre el citomegalovirus y la genética de la hipoacusia en los programas, así como los sistemas de control de las pérdidas de casos en el proceso. En futuras publicaciones se continuará con la revisión de los demás niveles y procesos de los programas (diagnóstico, tratamiento y seguimiento).

\section{Técnicas de cribado}

Para tener una audición normal es necesario que todo el sistema auditivo funcione correctamente. La mayor parte de las hipoacusias se producen por lesiones desde el oído externo hasta las células ciliadas externas que se encuentran en la cóclea. Para el estudio de la audición del recién nacido disponemos de una serie de pruebas que permiten conocer la integridad del sistema.

Actualmente se utilizan en nuestro medio dos pruebas en el momento del nacimiento: las Otoemisiones Acústicas con equipos automáticos (OEAa) y los Potenciales Evocados Auditivos automáticos (PEAa).

\section{- Otoemisiones acústicas}

Las otoemisiones constituyen una respuesta del normal funcionamiento de las células ciliadas externas. Representan una respuesta mecánica del oído interno considerándose un fenómeno pre neural porque se encuentran presentes, aunque se seccione el nervio auditivo y, además, invierten su polaridad junto con el estímulo.

Dado que la mayoría de las hipoacusias cursan con lesiones del oído externo, medio o de la cóclea, hasta las células ciliadas externas, las otoemisiones acústicas (OEA) se encuentran ausentes o reducidas en estos casos. Aunque existe un pequeño porcentaje de lesiones retrococleares que no se podrán diagnosticar por este método, las OEA siguen siendo útiles y ayudan a establecer la topografía de la lesión auditiva (Zubicaray et al., 2014: 1-15).

Las OEA con utilidad cínica son las provocadas, ya sean las transitorias o los productos de distorsión. Están presentes en el $98 \%$ de los pacientes normoyentes, desapareciendo cuando el umbral es mayor de $30 \mathrm{~dB}$ (Bonfils et al., 1990: 186-189).

Las Otoemisiones Acústicas Transitorias Evocadas (OEATE) son las que se utilizan con más frecuencia en los protocolos de cribado neonatal. Entre sus ventajas figura el bajo coste, la rapidez de ejecución y su alta sensibilidad, pero plantean algunos inconvenientes como la incapacidad para detectar lesiones posteriores a las células ciliadas externas, por lo que no se pueden diagnosticar las hipoacusias que son de origen retro- 
coclear $\mathrm{y}$, además, tienen una tasa de falsos positivos mayor que los $\mathrm{PEAa}$, sobre todo en los primeros días de vida (Boudewyns et al., 2016: 993-1000; Morant et al., 2014: 119-127).

Actualmente se utilizan para el cribado neonatal equipos de OEAa, que eliminan la subjetividad en la interpretación de los resultados.

\section{- Potenciales evocados auditivos}

Los PEAa, para identificar la audición correcta, se basan en un algoritmo matemático que elimina la subjetividad del explorador (Keohane et al., 2004: 112-116). Tienen una especificidad y sensibilidad muy alta, próxima al $100 \%$.

La ventaja de esta prueba estriba en que se trata de un método más sensible porque detecta las hipoacusias secundarias a neuropatía auditiva (hipoacusias retrococleares) (Duman et al., 2008: 1091-1095). Además, se puede realizar el primer día de vida y tiene un número menor de falsos positivos. Entre sus inconvenientes se puede señalar: el coste de los desechables, las interferencias eléctricas, por lo que es importante elegir bien dónde se lleva a cabo la prueba, el tiempo de realización y la menor precisión en el diagnóstico de frecuencias bajas, pudiendo disminuir la detección de las hipoacusias secundarias a patología del oído medio (Zubicaray et al., 2014: 1-15).

A la hora de plantear un cribado auditivo neonatal se pueden utilizar las OEAa, pero sabiendo que no se detectarán las sorderas retrococleares, lo que obliga a realizar además en los niños de alto riesgo PEAa o PEATC. Por otra parte, dado el mayor número de falsos positivos de las OEAa, se debe realizar el cribado en dos pasos para no remitir innecesariamente niños a la fase de diagnóstico.

El cribado basado en PEAa se puede realizar en un solo paso y además es posible diagnosticar la neuropatía auditiva, aunque hay que tener en cuenta que las hipoacusias de frecuencias bajas pueden pasar inadvertidas.

Es de esperar que, en un futuro, otras técnicas, como los potenciales evocados auditivos de estado estable (PEAee), puedan utilizarse en la fase de cribado (Mijares et al., 2015: 8-15).

\section{Pérdida de casos en el proceso}

La principal debilidad de los programas de cribado auditivo neonatal es la existencia de un alarmante porcentaje de niños que no han superado las pruebas de cribado iniciales y no son llevados por sus padres a las siguientes pruebas o revisiones. En un informe publicado por Centers for Disease Prevention and Control (CDC) se sugiere que cerca de la mitad de los casos cribados se pierden en el proceso o tienen una documentación incompleta de su situación (Bush et al. 2017: S1-13).

Estas altas tasas de pérdidas reducen la eficiencia de la detección y el tratamiento temprano de la hipoacusia. Como regla general, se admite que si las pérdidas superan el $20 \%$ se compromete la validez de los resultados 
del programa. En un meta-análisis llevado a cabo con 53 estudios publicados sobre la pérdida de casos en el proceso (Ravi et al., 2016: 29-36) se encontró que las tasas globales se sitúan en un $20 \%$ en estudios unicéntricos y en un $21 \%$ en los multicéntricos.

\subsection{Factores causantes}

Es importante conocer los factores que contribuyen a la pérdida de casos en el proceso de seguimiento, ya que ayudaría a formular nuevas regulaciones y a mejorar y cambiar los protocolos con el fin de aumentar la efectividad del programa de detección, diagnóstico y tratamiento precoz de la hipoacusia congénita. El principal factor reconocido en los distintos estudios sobre este problema es la falta de conocimiento sobre la importancia de completar el proceso diagnóstico de los niños que no superan el cribado de la hipoacusia. El siguiente factor en importancia es la distancia que separa el domicilio del niño de los centros donde se llevan a cabo las pruebas. Las obligaciones laborales y las actitudes desfavorables, tanto de los padres como de los profesionales sanitarios, se consideran también factores significativos.

La principal causa de la pérdida de los casos en el proceso es de origen parental, lo que resulta paradójico, teniendo en cuenta las altas tasas de satisfacción de los padres en las encuestas relativas a los programas de cribado neonatal de la hipoacusia (Nikolopoulos, 2015: 635-637). No obstante, también influyen otros factores como estar domiciliados en el medio rural, pertenecer a minorías étnicas o contar con escasos recursos económicos (Liu et al., 2008: e335-343).

Por tanto, y aunque en la mayoría de los programas están perfectamente delineados los circuitos de derivación durante el diagnóstico etiológico y audiológico del niño detectado en el cribado, resulta ser un proceso complejo y para muchos padres es difícil completarlo (DesGeorges, 2003: 89-93).

\subsection{Elementos de mejora}

A pesar de que algunos programas de detección precoz de la hipoacusia han intentado reducir las cifras de casos perdidos, en la actualidad no existe un método o abordaje estandarizado y basado en la evidencia. Sin embargo, la falta de adherencia a un proceso diagnóstico y terapéutico en otras áreas de la atención sanitaria sí ha sido objeto de intervención a través de los denominados "programas de navegación" para los pacientes.

La figura del 'navegador' es la de un profesional de la salud entrenado, que se dedica a asesorar y mitigar los factores personales o del medio, involucrados en la teoría social cognitiva (Bush et al., 2017: S1-13) para promover la adherencia a los procesos diagnósticos y terapéuticos. Estos profesionales asisten y orientan a los pacientes con el fin de facilitarles el cumplimiento con las citas de las consultas y las pruebas en los centros sanitarios. En el campo de la oncología, estos programas de navegación han sido especialmente exitosos para ayudar a los pacientes de origen socioeconómico deprimido, mejorando el cumplimiento con las citas médicas y obteniendo un diagnóstico en tiempo y forma, lo que se ha vinculado además con un ahorro significativo en el gasto sanitario.

Trasladadas estas experiencias al campo de la detección precoz de la hipoacusia, se ha llevado a cabo un ensayo prospectivo, controlado y randomizado (Bush et al., 2017: S1-13) en el que, comparado con la 
situación basal en la que no interviene "el navegador", se ha demostrado la eficacia de dicha intervención con los pacientes para disminuir las tasas de falta de adherencia en el proceso diagnóstico tras un fallo en las pruebas de cribado.

Las funciones del personal navegador deberían ser asumidas por un profesional del equipo interdisciplinar del programa de cribado de la hipoacusia, para lo que se precisará también de una base de datos fiable con el fin de conocer los casos que requieren ser contactados para captarlos. Además, dicha figura sería el enlace con el movimiento asociativo de familias, para que este pueda prestar el apoyo y la atención que las familias precisan, y actuaría, en su caso, como profesional de conexión con los servicios sociales.

Por tanto, las medidas más frecuentemente recomendadas para paliar las altas cifras de casos perdidos en el proceso incluyen la necesidad de contar con un equipo interdisciplinar comprometido, hacer campañas de concienciación pública en relación con la importancia de los programas de detección, diagnóstico y tratamiento precoz de la sordera infantil, así como la mejora en el mantenimiento de los necesarios sistemas de documentación y bases de datos.

\section{Infección congénita por citomegalovirus}

La infección congénita por citomegalovirus $(\mathrm{CMV}$ ) es muy común en todo el mundo, con una incidencia estimada en los países desarrollados que oscila entre 0,5 y $0,7 \%$ de todos los recién nacidos vivos (Fowler y Boppana, 2018: 149-154; Moresco et al., 2018: 88-91).

La incidencia en países en desarrollo es aún mayor (1-5\% de todos los nacimientos). La carga económica asociada a la CMVc es muy importante, puesto que los niños afectados requieren de un especial manejo terapéutico y educativo (Marsico y Kimberlin, 2017: 38).

\subsection{Diagnóstico de la CMVc}

El diagnóstico de la infección se basa en la detección del ADN de CMV mediante amplificación por reacción en cadena de la polimerasa (PCR), la cual presenta una alta sensibilidad y especificidad en una gran variedad de muestras biológicas como orina, saliva, sangre y otras (Guoyu et al., 2017: 376-386; Gantt et al., 2017: e267; De Vries et al., 2013: 113-117).

Las muestras preferidas para cribado en lactantes con CMVc son la orina y la saliva dado que en ellas se excretan grandes cantidades de virus, siendo más útiles que la sangre donde con frecuencia hay viremias bajas. Clásicamente se ha recogido la orina por bolsa con una sensibilidad del $100 \%$ y especificidad del $99 \%$, por lo que una sola muestra negativa ya es suficiente para descartar la infección y una positiva recogida antes de los 21 días la confirmaría (Luck et al., 2017: 1205-1213). Sin embargo, recoger una muestra de orina por bolsa tiene varias limitaciones, que pueden ser obviadas con garantías realizando la recogida de orina mediante algodones en el pañal (Ross et al., 2015: 903-905). 
La opción más factible es la recogida de una muestra de saliva (en fresco o en seco), pero también tiene sus limitaciones porque es menos sensible y sobre todo porque se puede contaminar con la ingesta de la leche materna, que podría estar infectada con CMV en un 0,03 a 0,14\% de casos. Un resultado positivo siempre debe ser confirmado con una muestra de orina (Hilditch et al., 2018: 988-992; Kummer y Marcrum, 2018: 2026; Rawlinson et al., 2017: e177-188; Cardoso et al., 2015: 206-207; Gunkel et al., 2014: 61-64; Boppana et al., 2011: 2111-2118; Lawrence, 2006: 99-107).

Si el niño tiene más de tres semanas de vida, la PCR en orina, saliva o sangre no sería definitiva, ya que puede deberse a una infección, tanto congénita como adquirida, y habría que recurrir a la PCR en el papel secante de la prueba de cribado metabólico para poder confirmarlo. Un resultado positivo confirmaría la infección, aunque uno negativo no la descarta dada la peor sensibilidad de este método (Moteki et al., 2018: 708-712; Vives-Oñós et al., 2018: 520-524; Ross et al., 2017: 57-61; Koontz et al., 2015: 95-99; Boppana et al., 2010: 1375-1382; Choi et al., 2009: 1095-1098).

Esta prueba puede ser muy útil en el diagnóstico retrospectivo de CMVc y puede proporcionar información sobre la etiología de la hipoacusia. Un análisis retrospectivo demostró que el $26 \%$ de los pacientes que presentaban hipoacusias idiopáticas en la infancia tenían CMV detectable en la prueba de cribado metabólico (Meyer et al., 2017: 565-570). La serología del CMV no se considera una técnica útil para el diagnóstico de la infección congénita (Badia, 2014: 356-366).

Estudios recientes han señalado a la CMVc como una de las causas más importantes de hipoacusia congénita y también postnatal, solo por detrás de las de origen genético, puesto que se detecta en un alto porcentaje de los niños con hipoacusia comprobada. Hay estudios que identifican la CMVc como la causa, aproximadamente, del $20 \%$ de los casos de hipoacusia neurosensorial congénita, ascendiendo al $25 \%$ de los casos a la edad de cuatro años (Kummer y Marcrum, 2018: 20-26; Nance et al., 2006: 221-225; Morton y Nance, 2006: 2151-2164).

\subsection{CMVc asintomática y sintomática}

Habitualmente la infección CMVc se clasifica en sintomática o asintomática en el momento del nacimiento. Los síntomas en el recién nacido son de diversa índole, pero en la práctica solo se detectarán signos o síntomas de CMVc en el examen neonatal de rutina en alrededor del $13 \%$ de casos, por lo que la gran mayoría permanece sin diagnosticar a esta edad, siendo catalogados como asintomáticos (Fowler y Boppana, 2018: 149-154; Kummer y Marcrum, 2018: 20-26; Lim y Lyall, 2017: S89-94; Williams et al., 2015: F501-506).

Datos recientes demuestran que, a largo plazo, un alto porcentaje de niños asintomáticos (25\%) desarrollarán hipoacusia (Lanzieri et al., 2018: 736-744; Lanzieri et al., 2017a: 875-880). En números globales, la mayoría de niños con hipoacusia por CMVc $(58 \%)$ habrán sido catalogados como niños asintomáticos (Bartlett et al., 2017: e1938; López et al., 2017: e20171517; Goderis et al., 2014: 972-982).

Se considera que un niño es sintomático si presenta signos o síntomas típicos en la esfera hematológica, oftalmológica, auditiva o neurológica, entre otras (Luck et al., 2017: 1205-1213). 
La sospecha diagnóstica obliga a realizar estudios complementarios de diversa índole. Para el estudio auditivo se recomienda utilizar los potenciales evocados (PEA), dado que la pérdida auditiva típica de la CMVc puede ser coclear y/o retrococlear (Lanzieri et al., 2018: 736-744; Lim y Lyall, 2017: S89-94; Luck et al., 2017: 1205-1213).

\subsection{Pérdida de audición asociada a CMVc}

En los niños sintomáticos la hipoacusia es la secuela más frecuente. Un 30-65\% tendrá una hipoacusia que puede ser detectada al nacer; pero en un $18-30 \%$ de los casos su aparición será posterior, totalizando un $74 \%$ a los 18 años. La hipoacusia de inicio diferido se puede observar tanto en pacientes asintomáticos como sintomáticos, aunque en diferentes proporciones (9-18\%) (Kummer y Marcrum, 2018: 20-26).

Además, es a menudo progresiva (18-63\% de casos), llegando a profunda en el $78 \%$ de ellos, a lo largo de los primeros seis años de vida (Goderis et al., 2016: 110-115; Goderis et al., 2014: 972-982).

Esta hipoacusia también suele ser unilateral, sobre todo en pacientes asintomáticos (57\%), en quienes puede ser la única manifestación, y es habitualmente grave. Con cierta frecuencia se presenta de forma asimétrica. Otra particularidad es que puede ser fluctuante (20-24\%) en un solo oído o en algunas frecuencias (Kim et al., 2018: 1-8).

La pérdida de audición asociada a la CMVc no será detectada a menos que la infección se identifique durante el embarazo, por clínica neonatal o en forma de cribado del CMV más o menos sistemático. Solo en torno al $10 \%$ de los niños con pérdida de audición al nacer, relacionada con CMVc, se diagnostica por signos clínicos de la infección (Kummer y Marcrum, 2018: 20-26).

En un amplio estudio multicéntrico en los Estados Unidos para la detección de CMVc, se encontró que los niños infectados tienen siete veces más probabilidades de no superar el cribado auditivo (Fowler et al., 2017: e20162128).

La mitad $(52 \%)$ de todos los casos de pérdida de audición asociada con CMVc está presente desde el nacimiento, de ahí el interés del cribado del CMVc dirigido/selectivo a los recién nacidos que no pasan las pruebas de evaluación auditiva (Park et al., 2014: 2624-2629; Choi et al., 2009: 1095-1098; Stehel et al., 2008: 970-975). Además, una parte importante de los niños hipoacúsicos infectados por CMVc se diagnosticarán exclusivamente por no haber superado el cribado auditivo neonatal.

Varios estudios concluyen que del 5 al $6 \%$ de recién nacidos no superaron el cribado auditivo a causa de la infección CMVc (Rawlinson et al., 2018: 110-115; Ari-Even Roth et al., 2017: F519-524; Diener et al., 2017: e20160789; Fowler et al., 2017: e20162128).

Recientemente se han publicado datos que demuestran que un cribado de CMV en recién nacidos que no han superado el cribado auditivo neonatal es coste-efectivo, permitiendo un ahorro de más de un $50 \%$ del gasto (Vancor et al., 2019: 55-59; Williams et al., 2015: F501-506; Williams et al., 2014: F230-236; Kadambari et al., 2013: 928-933). 


\subsection{Implicaciones en el cribado auditivo y tratamiento}

El diagnóstico de la infección congénita tiene especial interés en los niños que no superan todo el proceso de cribado auditivo antes de las 2-3 semanas de vida porque así se iniciaría ya su diagnóstico etiológico y su seguimiento en tiempo y forma (Luck et al., 2017: 1205-1213; Yamaguchi et al., 2017: e013810).

Como ya se ha comentado, el tiempo límite para diagnosticar la infección congénita con seguridad son las dos o tres semanas de vida postnatal. La positividad del CMV permitiría así hacer el diagnóstico seguro de infección congénita (Botet et al., 2015: 69; Escosa-García et al., 2015: 70-71). Este hecho es determinante en aquellos programas de cribado de hipoacusia que estudian la infección congénita por CMV cuando el neonato presenta un cribado auditivo alterado (cribado selectivo), puesto que obliga a establecer un mecanismo diagnóstico en esa ventana temporal. Por tanto, obliga a realizar la segunda prueba de cribado (en aquellos programas con dos pasos) alrededor de los 15 días de vida, para así hacer posible la detección de la enfermedad congénita y la valoración del inicio del tratamiento, si procede, antes del mes de vida, como parece ser recomendable (Rawlinson et al., 2018: 110-115). Para ello, es necesario recordar que las muestras de saliva positivas se deben confirmar con otra muestra de orina en el tiempo descrito, lo que debe tenerse en cuenta a la hora de diseñar los cambios a realizar en los protocolos.

Por otra parte, no hay que perder de vista que un gran estudio multicéntrico (Kummer y Marcrum, 2018: 2026; Rawlinson et al., 2018: 110-115) demostró que, aunque la infección congénita está presente en el $6 \%$ de los niños que fallan en el cribado auditivo para recién nacidos, el cribado selectivo solo pudo identificar el $57 \%$ de los niños con hipoacusia relacionada con la CMVc.

Estas limitaciones del cribado selectivo apoyan la necesidad de plantear un cribado universal. Este permitiría captar en el tiempo adecuado aquellos neonatos infectados pero asintomáticos y que tienen una primera determinación auditiva normal, con riesgo de presentar una hipoacusia posterior. El cribado universal estaría justificado dada la prevalencia de la infección y la posibilidad de mejorar el pronóstico con un manejo, seguimiento y tratamiento adecuado (Fowler et al., 2017: e20162128; Toumpas et al., 2015: 541-544).

Un estudio reciente ha demostrado que tanto el cribado dirigido como el universal son coste-efectivos (Fowler et al., 2017: e20162128; Kadambari et al., 2015: 1117-1121; Kimberlin et al., 2015: 933-943; Barkai et al., 2014: 361-366; Cannon et al., 2014: 291-307).

A diferencia de la infección congénita por CMV, la infección adquirida en el neonato y lactante no parece asociarse a sordera ni a alteraciones en el neurodesarrollo a largo plazo, de ahí la importancia del diagnóstico preciso del momento de la infección, con la detección por PCR al nacer o en la sangre seca de la prueba de cribado metabólico (Smiechura et al., 2014: 303-307; Nuñez-Ramos et al., 2013: 93-96; Botet et al., 2015: 69).

El diagnóstico de la infección congénita tiene especial interés en los niños que no superan todo el proceso de cribado auditivo y son remitidos al ORL para confirmación antes de las 2-3 semanas de vida porque la mayoría de estudios han concluido que el inicio del tratamiento para la CMVc podría ser efectivo, sobre todo en aquellos con hipoacusia moderada, si se inicia antes del mes de vida y se prolonga varios meses, al menos entre seis y doce meses (Bilavsky et al., 2016: 433-438; Kimberlin et al., 2015: 933-943; Amir et al., 2010: 1061-1067; Kimberlin et al., 2003: 16-25). 
El tratamiento con Valganciclovir (VGC) está recomendado en niños sintomáticos con infección congénita demostrada y con afectación del sistema nervioso central o de los órganos diana (médula ósea, hígado) siempre que sea grave. En el caso de niños asintomáticos con hipoacusia aislada, se recomienda no tratar debido a la ausencia de evidencia, pero se aconseja valorar cada caso de forma individual.

La investigación de la infección CMVc también está indicada en los lactantes y niños con hipoacusia comprobada por medio de muestras recogidas en el período neonatal (por ejemplo, la muestra del talón).

Respecto a iniciar el tratamiento en niños con hipoacusia por CMVc mayores de un mes, la European Society for Paediatric Infectious Diseases (ESPID) (Luck et al., 2017: 1205-1213) concluyó que era aceptable tratar si existe deterioro progresivo de la audición (Amir et al., 2014: 444-448; Del Rosal et al., 2012: 72-74; Baquero et al., 2009: 535-547).

Los niños asintomáticos no deben ser tratados según las recomendaciones de los dos comités de expertos que han estudiado el tema (Rawlinson et al., 2017: e177-188; Luck et al., 2017: 1205-1213).

Aunque se discuta el tratamiento farmacológico, el simple conocimiento de la infección permite el seguimiento prolongado de estos niños y la posibilidad de un diagnóstico auditivo adecuado en un tiempo que permita el tratamiento audiológico más indicado de forma precoz (Hilditch et al., 2018: 988-992). De hecho, el conocimiento de la infección CMVc puede hacer variar al clínico su decisión sobre la colocación de un implante coclear de forma precoz, debido a la gran probabilidad de progresión que presenta esta enfermedad (Lanzieri et al., 2017b: e20162610).

Dado que la sordera por CMV se presenta en niños sintomáticos y asintomáticos, siendo fluctuante y con frecuencia postnatal, se recomienda un seguimiento periódico frecuente durante los primeros dos años de vida, siendo este el período de mayor riesgo para el desarrollo de pérdida auditiva asociada a CMVc y un período crítico para el desarrollo del lenguaje. Aunque en general debe seguirse a estos niños durante al menos seis años, con revisiones más frecuentes en los más afectados.

\section{Perspectivas de futuro: cribado combinado}

Actualmente los programas de cribado tienen limitaciones para detectar las hipoacusias leves o moderadas (Johnson et al., 2005:663-672), así como para detectar las hipoacusias de inicio tardío o las que progresan tras el nacimiento. Muchas de estas tienen en su etiología la infección por CMVc y/o alteraciones genéticas. Debido a ello, estos niños no se beneficiarán de la mejoría pronóstica que confiere la detección y tratamiento temprano de las hipoacusias (Young et al., 2011: 230-234).

Hasta un $60 \%$ de las hipoacusias neurosensoriales congénitas o de inicio precoz están producidas por factores genéticos, apareciendo habitualmente en ausencia de historia familiar de hipoacusia (Hilgert et al., 2009:189-196). Las alteraciones más frecuentemente encontradas se localizan en el gen GJB2 (conexina 26), que es la variante más frecuente a nivel mundial, seguido del SCL26A4, que es responsable del sín- 
drome de Pendred, cuyas mutaciones se asocian con un $3 \%$ de sordera neonatal, aunque este porcentaje aumenta significativamente con el paso de los años y parece asociarse con un acueducto del vestíbulo dilatado (Morton y Nance, 2006: 2151-2164; Pryor et al., 2005:159-165). También son relativamente frecuentes las mutaciones del gen mitocondrial MTRNR1 que se pueden manifestar con una hipoacusia inducida por tratamiento con aminoglucósidos (Del Castillo et al., 2002: 243-249).

\subsection{Etiología e incidencia}

Aunque hay variaciones entre las distintas poblaciones, estudios a gran escala demuestran que se repiten las etiologías e incidencias, como es el caso de un estudio reciente sobre 142.417 neonatos de China, donde el alelo relacionado con hipoacusia más frecuentemente encontrado fue el 235 de IC variante en el gen GJB2. En total, 4289 recién nacidos (3,01\%) tenían alteraciones en al menos un alelo de alguno de los genes estudiados (Hao et al., 2018: e0195740). Dado que las causas genéticas producen un alto porcentaje de las hipoacusias neurosensoriales y que muchas de ellas no pueden ser detectadas en el cribado auditivo universal, existen estudios que combinan el cribado auditivo convencional con un cribado genético en recién nacidos para las mutaciones más frecuentes causantes de hipoacusia. Wang et al. (2011) realizaron un cribado genético, combinado con el convencional (con OEA y PEAa), para la determinación de los tres genes más frecuentemente asociados con la hipoacusia en más de 14.000 recién nacidos, concluyendo que al añadir el cribado genético se podría mejorar la detección de los pacientes con alteraciones auditivas (Wang et al., 2011: 535-542).

Recientemente, Wu et al. (2017) encontraron un 1,6\% de dichas alteraciones genéticas en la población estudiada (5173 recién nacidos). Lo más destacable fue que el 56,1\% había pasado el cribado auditivo convencional (Wu et al., 2017: 6-12). En otro estudio, realizado también en China, se combinó el cribado convencional con la determinación de veinte mutaciones en los cuatro genes más frecuentemente asociados con hipoacusia neurosensorial, por medio de la muestra de sangre de talón, obtenida para realización de la prueba de cribado de enfermedades endocrino-metabólicas, confirmando los resultados comentados anteriormente: un total de 129 niños (1,38\%) presentaron hipoacusia detectada mediante las OEA. El cribado genético determinó que 348 individuos $(3,74 \%)$ tenían al menos una mutación en uno de los alelos. Una abrumadora mayoría de recién nacidos con estas alteraciones genéticas había pasado el cribado auditivo con OEA, pero tenían riesgo de hipoacusia tardía (Peng et al., 2016: 603-608).

\subsection{Utilidad del cribado combinado}

La utilidad del cribado combinado ha sido demostrada también por otro estudio reciente (Sun et al., 2015: 766-770), en el que se investigó la tasa de detección de casos de hipoacusia mediante el cribado auditivo, genético o combinado en 11.046 recién nacidos. Encontraron que la prueba combinada de audición y genética detectó un 5,29\% de casos, cuando la auditiva de forma aislada había identificado un 0,81 \% y la genética aislada un 4,64\%. Está en proyecto la realización de estudios genéticos para detectar mutaciones de genes relacionados con la hipoacusia congénita en gestantes, lo que podría facilitar su detección precoz (Fang et al., 2017: 1452-1455).

Así pues, la utilización del cribado genético, junto con el cribado auditivo en los recién nacidos, es beneficioso para mejorar la tasa de detección de niños en riesgo, así como para la identificación temprana de los 
mismos, antes del desarrollo del lenguaje. Se podría prevenir también el desarrollo de hipoacusia evitando el uso de aminoglucósidos en pacientes portadores de mutaciones sensibles a los mismos y toda la información obtenida se podría utilizar para el consejo genético.

En un futuro el cribado de la CMVc se puede integrar con el estudio genético, además del cribado auditivo, a modo de panel diagnóstico de sordera. Este enfoque permitiría obviar las limitaciones del cribado auditivo neonatal actual y detectaría todos los niños con riesgo auditivo presente o futuro. Hay que recordar que la infección por CMV no excluye la posibilidad de presentar de forma simultánea una alteración genética relacionada con la pérdida auditiva, como han demostrado algunos estudios (Teek et al., 2013: 419-428; Lim et al., 2013: 209-215; Karltorp et al., 2012: e357-362; Schimmenti et al., 2011: 1006-1010).

Concretamente, en el estudio de Lu et al. (2018), se realizó un análisis prospectivo sobre 1716 recién nacidos. De ellos, resultó positivo el cribado genético en veinte $(1,2 \%)$ y el cribado de CMV en tres $(0,2 \%)$. Lo interesante es que doce de los veinte recién nacidos que tuvieron un cribado genético positivo $(60 \%)$ y los tres positivos para CMV (100\%), superaron el cribado auditivo al nacer. A los tres meses se confirmó la pérdida auditiva en seis de los veinte pacientes con genética positiva (30\%). Este estudio ratifica que realizar cribado auditivo, genético y de CMV ayuda a detectar casos que no serían identificados mediante los programas habituales de cribado auditivo (Lu et al., 2018: 144-150). Por tanto, plantear un cribado integrado y completo con estudio auditivo, genético y de la infección CMVc es factible y se obtienen buenos resultados, pero se necesitan más estudios de coste-efectividad.

El cribado genético combinado descrito permitiría un seguimiento adecuado y un tratamiento audiológico y/o farmacológico precoz en los niños afectos, en espera de un futuro cercano en el que, si conocemos los defectos genéticos a los que nos enfrentamos de forma precoz, además de realizar un consejo genético preciso, podremos corregirlos gracias a técnicas como CRISP-Cas9. Existen ya estudios que demuestran que esto es posible en relación a la hipoacusia en animales, por lo que este enfoque seguro que mejorará el pronóstico final de los niños con hipoacusia (Zou et al., 2015: 102-108; Gao et al., 2018: 217-221).

\section{Recomendaciones CODEPEH 2018}

La amplia implantación de los programas de cribado neonatal de la hipoacusia puede llevar a pensar que, descartada en ese momento, ya no afectará al niño a lo largo de su desarrollo. Esta es una falsa creencia que puede perjudicar seriamente el futuro de esos niños, puesto que factores postnatales, no infrecuentes, pueden originar una pérdida auditiva. La prevalencia podría pasar del 2,52 por mil al nacer (cualquier tipo y grado de hipoacusia) al 3,64 por mil en la etapa de la educación primaria (Watkin y Baldwin, 2012: 519-528; Watkin y Baldwin, 2011: 62-66).

Se ha visto que, aún en los cribados con altas sensibilidades en las pruebas neonatales, estas solo identifican el 56-59\% de niños en edad escolar con sordera. Así, hasta uno de cada diez niños con hipoacusia sería detectado por revisiones postnatales, a pesar de tener protocolos de cribado bien establecidos. La 
prevalencia global de una hipoacusia de aparición tardía es del 10\% sobre todas las hipoacusias de la infancia, pudiendo llegar al 20\% (Benito, 2013: 330-342; Georgalas et al., 2008: 1299-1304). Todo ello sugiere la necesidad de protocolos diagnósticos que permitan identificar los casos de sordera postneonatal.

Gran parte de las sorderas de inicio tardío en la infancia parecen ser debidas a defectos genéticos, infección congénita por citomegalovirus ( $\mathrm{CMV}$ ) o trastornos del acueducto del vestíbulo, lo que obliga al estudio genético, a descartar una infección por citomegalovirus (CMV) y a la realización de estudios de imagen complementarios (Alford, 2014: 347-355).

Por todo ello, la CODEPEH considera necesario formular unas nuevas recomendaciones para la actualización de los programas de cribado auditivo infantil, en relación con la fase de detección, adoptando los cambios expuestos en el presente documento (Figura 1):

1. Las OEAa y los PEAa siguen siendo las técnicas recomendadas para el cribado auditivo neonatal.

2. Los programas de cribado auditivo tienen que mantenerse en alerta ante el porcentaje de casos que se pueden perder en el proceso de detección, diagnóstico y tratamiento precoz, habilitando las medidas oportunas para evitar este riesgo.

3. El movimiento asociativo de familias ha de ser un punto de apoyo a lo largo de todo el proceso.

4. Debe llevarse a cabo la detección del CMV por PCR en todos los neonatos que no superen el cribado auditivo neonatal bilateral.

5. El diagnóstico de la infección CMVc se debe hacer antes de las tres semanas de vida y su tratamiento, en caso necesario, se debe iniciar antes del mes. Para ello hay que adaptar los protocolos de cribado actuales, y así se podrá aplicar también un protocolo de seguimiento del riesgo auditivo ajustado a las peculiaridades del CMV (hipoacusia unilateral, progresiva, fluctuante, asimétrica...).

6. Además del seguimiento por ORL, en los niños con CMVc es necesario un estudio completo por parte de los servicios de Pediatría y Oftalmología.

7. En un futuro próximo se debería plantear el cribado universal de la infección CMVc, dado que el estudio selectivo identifica solo el $57 \%$ de los niños infectados que presentarán hipoacusia en la infancia, siendo ambos coste-efectivos.

8. El cribado auditivo, el estudio genético y el estudio del citomegalovirus se deberían integrar en un mismo protocolo, cuando sea posible, para lograr el completo diagnóstico y tratamiento precoz de la hipoacusia infantil. 


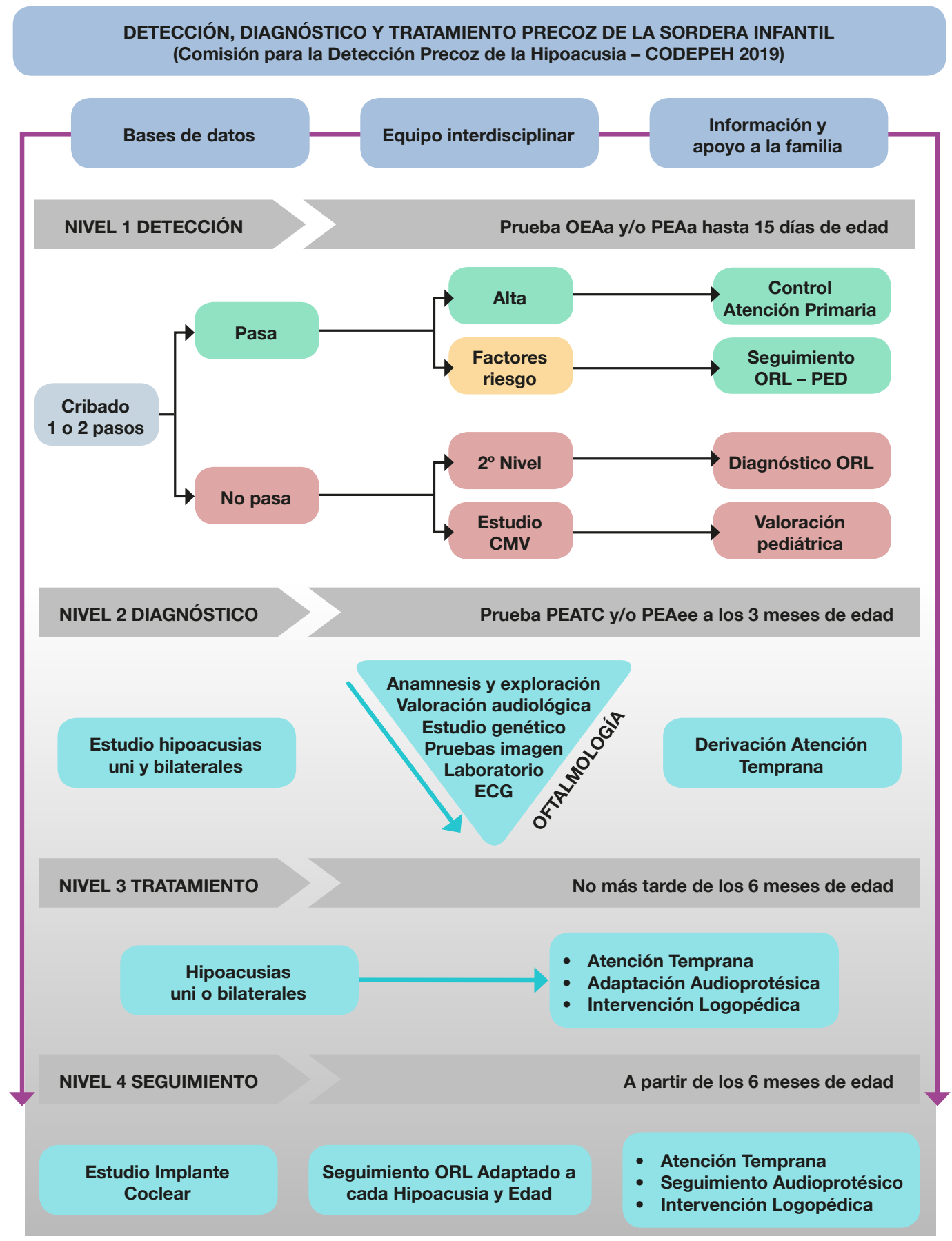

@ CODEPEH 2018

OEAa: Otoemisiones acústicas automáticas. PEAa: Potenciales Evocados Auditivos automatizados. PEATC: Potenciales Evocados Auditivos de Tronco Cerebral. PEAee: Potenciales Evocados Auditivos de estado estable.

Fuente: elaboración propia. 


\section{Referencias bibliográficas}

Alford, R. L. (2014): "American College of Medical Genetics and Genomics guideline for the clinical evaluation and etiologic diagnosis of hearing loss". Genet Med., 16 (4): 347-355.

Amir, J. et al. (2014): "Treatment of late-onset hearing loss in infants with congenital cytomegalovirus infection". Clin Pediatr, 53: 444-448.

Amir, J. et al. (2010): "Treatment of symptomatic congenital cytomegalovirus infection with intravenous ganciclovir followed by long-term oral valganciclovir". Eur J Pediatr, 169: 1061-1067.

Ari-Even Roth, D. et al. (2017): "Contribution of targeted saliva screening for congenital CMV-related hearing loss in newborns who fail hearing screening" Arch Dis Child Fetal Neonatal Ed, 102: F519-524.

Badia, J. (2014): “Infecciones congénitas”. Pediatr Integral, 18: 356-366.

Baquero, F. et al. (2009): "Documento de consenso de la Sociedad Española de Infectologia Pediátrica sobre el diagnóstico y el tratamiento de la infección congénita por citomegalovirus”. An Pediatr (Barc), 71: 535-547.

Barkai, G. et al. (2014): "Universal neonatal cytomegalovirus screening using saliva. Report of clinical experience". J ClinVirol, 60: 361-366.

Bartlett, A. W. et al. (2017): "Hearing and neuro developmental outcomes for children with asymptomatic congenital cytomegalovirus infection: A systematic review". Rev Med Virol, 27: e1938.

Benito, J. I. (2013): “Hipoacusia: identificación e intervención precoces”. Pediatr Integral, 17: 330-342.

Bilavsky, E. et al. (2016): "Hearing outcome of infants with congenital cytomegalovirus and hearing impairment". Arch Dis Child, 101: 433-438.

Bonfils, P. et al. (1990): "Evoked otoacoustic emissions in newborn hearing screening”. Laryngoscope, 100: 186-189.

Boppana, S. B. et al. (2011): "National Institute on Deafness and Other Communication Disorders CHIMES Study. Saliva polymerase-chain-reaction assay for cytomegalovirus screening in newborns". N Engl J Med, 364: 2111-2118.

Boppana, S. B. et al. (2010): "Dried blood spot real-time polymerase chain reaction assays to screen newborns for congenital cytomegalovirus infection". JAMA, 303: 1375-1382.

Botet, F. et al. (2015): "Cribado universal de infección por citomegalovirus en prematuros de menos de 1.500 g". An Pediatr, 83: 69.

Boudewyns, A. et al. (2016): "Auditory neuropathy spectrum disorder (ANSD) in referrals from neonatal hearing screening at a well-baby clinic". Eur J Pediatr, 175 (7): 993-1000.

Bruijnzeel, H. et al. (2016): "A systematic review to define the speech and language benefit of early $(<12$ Months) pediatric cochlear implantation". Audiol Neurotol, 21: 113-126.

Bush, et al. (2017): "Promotion of early pediatric hearing detection through patient navigation: a randomized controlled clinical trial”. Laryngoscope, 127: S1-13.

Cannon, M. J. et al. (2014): "Universal newborn screening for congenital CMV infection: what is the evidence of potential benefit?". Rev Med Virol, 24: 291-307. 
Cardoso, E. S. et al. (2015): "The use of saliva as a practical and feasible alternative to urine in large-scale screening for congenital cytomegalovirus infection increases inclusion and detection rates". Rev Soc Bras Med Trop, 48: 206-207.

Ching, T. Y. C. et al. (2013): "Outcomes of early- and late-identified children at 3 years of age: findings from a prospective population-based study". Ear Hear, 34: 535-552.

Choi, K. Y. et al. (2009): "Detection of cytomegalovirus DNA in dried blood spots of Minnesota infants who do not pass newborn hearing screening". Pediatr Infect Dis J, 28: 1095-1098.

De Vries, J. J. et al. (2013): "Cytomegalovirus DNA detection in dried blood spots and perilymphatic fluids from pediatric and adult cochlear implant recipients with prelingual deafness". J ClinVirol, 56: 113-117.

Del Castillo, I. et al. (2002): "A deletion involving the connexin 30 gene in nonsyndromic hearing impairment". $N$ Engl J Med, 346 (4): 243-249.

Del Rosal, T. et al. (2012): "Treatment of symptomatic congenital cytomegalovirus infection beyond the neonatal period". J ClinVirol, 55: 72-74.

DesGeorges, J. (2003): "Family perceptions of early hearing, detection, and intervention systems: listening to and learning from families". Ment Retard Dev Disabil Res Rev, 9: 89-93.

Diener, M. L. et al. (2017): "Outcomes from a hearingtargeted cytomegalovirus screening program". Pediatrics, 139: e20160789.

Duman, K. et al. (2008): "Incidence of auditory neuropathy among the deaf school students". Int J Pediatr Otorhinolaryngol, 72 (7): 1091-1095.

Escosa-García, L. et al. (2015): “Cribado de citomegalovirus en prematuros menores de 1.500 g. Comité Científico del Registro Estatal de Infección Congénita por Citomegalovirus”. An Pediatr, 83: 70-71.

Fang, Y. et al. (2017): "Application of gene detection technique in the antenatal diagnosis of hereditary hearing loss". Eur Rev Med Pharmacol Sci, 21: 1452-1455.

Fowler, K. B. y Boppana, S. B. (2018): “Congenital cytomegalovirus infection”. Semin Perinatol, 42: 149-154.

Fowler, K. B. et al. (2017): "A targeted approach for congenital cytomegalovirus screening within newborn hearing screening”. Pediatrics, 139 (2): e20162128.

Gantt, S. et al. (2017): "In reference to should infants who fail their newborn hearing screen undergo cytomegalovirus testing?". Laryngoscope, 128: e267.

Gao, X. et al. (2018): "Treatment of autosomal dominant hearing loss by in vivo delivery of genome editing agents". Nature, 553 (7687): 217-221.

Georgalas, C. et al. (2008): "Screening for hearingloss and middle-ear effusion in school-age children, using transient evokedoto acoustic emissions: a feasibility study”. J Laryngol Otol, 122: 1299-1304.

Goderis, J. et al. (2016): "Hearing in children with congenital cytomegalovirus infection: results of a longitudinal study". J Pediatr, 172: 110-115.e2.

Goderis, J. et al. (2014): "Hearing loss and congenital CMV infection: a systematic review”. Pediatrics, 134: $972-982$.

Gunkel, J. et al. (2014): "Urine is superior to saliva when screening for postnatal CMV infections in preterm infants". J ClinVirol, 61: 61-64. 
Guoyu, L. et al. (2017): "Detection of congenital cytomegalovirus in newborns using nucleic acid amplification techniques and its public health implications". Virologica Sinica, 32: 376-386.

Hao, Z. et al. (2018): "Large scale newborn deafness genetic screening of 142.417 neonates in Wuhan, China". PLoS One, 13 (4): e0195740.

Hilditch, C. et al. (2018): "Does screening for congenital cytomegalovirus at birth improve longer term hearing outcomes?". Arch Dis Child, 103: 988-992.

Hilgert, N. et al. (2009): "Forty-six genes causing nonsyndromic hearing impairment: which ones should be analyzed in DNA diagnostics?". Mutat Res, 681:189-196.

Johnson, J. L. et al. (2005): "A multicenter evaluation of how many infants with permanent hearing loss pass a twostage otoacustic emissions/automated auditory brainstem response newborn hearing protocol". Pediatrics, 116: 663-672.

Kadambari, S. et al. (2015): "Evaluating the feasibility of integrating salivary testing for congenital CMV into the Newborn Hearing Screening Programme in the UK”. Eur J Pediatr, 174: 1117-1121.

Kadambari S, et al. (2013): "Clinically targeted screening for congenital CMV. Potential for integration into the National Hearing Screening Programme". Acta Paediatr, 102: 928-933.

Karltorp, E. et al. (2012): "Congenital cytomegalovirus infection: a common cause of hearing loss of unknown aetiology”. Acta Paediatr, 101 (8): e357-362.

Kennedy, C. R. et al. (2006): "Language ability after early detection of permanent childhood hearing impairment". N Engl J Med, 354: 2131-2141.

Kennedy, C. R. et al. (2005): "Universal newborn screening for permanent childhood hearing impairment: an 8-year follow-up of a controlled trial”. Lancet, 366: 660-662.

Kennedy, C. R. (1999): "Controlled trial of universal neonatal screening for early identification of permanent childhood hearing impairment: coverage, positive predictive value, effect on mothers and incremental yield. Wessex Universal Neonatal Screening Trial Group". Acta Paediatr Suppl, 88 (432): 73-75.

Keohane, B. M. et al. (2004): "Clinical evaluation of the vector algorithm for neonatal hearing screening using automated auditory brainstem response". J Laryngol Otol, 118 (2): 112-116.

Kim, B. J. et al. (2018): "Characterization of detailed audiological features of cytomegalovirus Infection: a composite cohort study from groups with distinct demographics". Biomed Res Int, 5: 1-8.

Kimberlin, D. W. et al. (2015): "National Institute of Allergy and Infectious Diseases Collaborative Antiviral Study Group. Valganciclovir for symptomatic congenital cytomegalovirus disease”. N Engl J Med, 372: 933-943.

Kimberlin, D. W. et al. (2003): "National Institute of Allergy and Infectious Diseases Collaborative Antiviral Study Group. Effect of ganciclovir therapy on hearing in symptomatic congenital cytomegalovirus disease involving the central nervous system: a randomized, controlled trial”. J Pediatr, 143: 16-25.

Koontz, D. et al. (2015): "Evaluation of DNA extraction methods for the detection of Cytomegalovirus in dried blood spots". J ClinVirol, 66: 95-99.

Korver, A. M. H. et al. (2010): "Newborn hearing screening vs later hearing screening and developmental outcomes in children with permanent childhood hearing impairment”. JAMA, 304: 1701-1708. 
Kummer, P. y Marcrum, S. C. (2018): "Potential benefit of selective CMV testing after failed newborn hearing screening". Int. J. Neonatal Screen, 4: 20-26.

Lanzieri, T. M. et al. (2018): "Congenital cytomegalovirus longitudinal study group. hearing trajectory in children with congenital cytomegalovirus infection”. Otolaryngol Head Neck Surg, 158: 736-744.

Lanzieri, T. M. et al. (2017a): "Long-term outcomes of children with symptomatic congenital cytomegalovirus disease”. J Perinatol, 37:875-880.

Lanzieri, T. M. et al. (2017b): "Hearing loss in children with asymptomatic congenital cytomegalovirus infection. Congenital Cytomegalovirus Longitudinal Study Group”. Pediatrics, 139 (3): e20162610.

Lawrence, R. M. (2006): “Cytomegalovirus in human breast milk: risk to the premature infant”. Breastfeed Med, 1 : 99-107

Lim, B. G. et al. (2013): "Utility of genetic testing for the detection of late-onset hearing loss in neonates". Am J Audiol, 22: 209-215.

Liu, C. L. et al. (2008): "Evaluating loss to follow-up in newborn hearing screening in Massachusetts". Pediatrics, 121 (2): e335-343.

Lim, Y. y Lyall, H. (2017): "Congenital cytomegalovirus. Who, when, what, with and why to treat?". J Infect, 1: S89-94.

López, A. S. et al. (2017): "Congenital cytomegalovirus longitudinal study group. Intelligence and Academic Achievement with Asymptomatic Congenital Cytomegalovirus Infection”. Pediatrics, 140 (5): e20171517.

Lu, C. Y. et al. (2018): "Concurrent hearing, genetic, and cytomegalovirus screening in newborns, Taiwan". J Pediatr, 199: 144-150.

Luck, S. E. et al. (2017): "Congenital cytomegalovirus: A european expert consensus statement on diagnosis and management”. Pediatr Infect Dis J, 36: 1205-1213.

Marsico, C. y Kimberlin, D. W. (2017): "Congenital cytomegalovirus infection: advances and challenges in diagnosis, prevention and treatment". Ital J Pediatr, 43: 38.

Meyer, L. et al. (2017): "Analysis of archived newborn dried blood spots (DBS) identifies congenital cytomegalovirus as a major cause of unexplained pediatric sensorineural hearing loss". Am J Otolaryngol, 38: 565-570.

Mijares, E. et al. (2015): "Hearing screening using auditory steady state responses obtained by simultaneous airand bone-conduction stimuli". Acta Otorrinolaringol Esp, 66: 8-15.

Morant, A. et al. (2014): “Otoemisiones”. En Manrique, M. y Marco, J. (coords.): Audiología, Ponencia Oficial de la Sociedad Española de Otorrinolaringología y Patología Cérvico-Facial. Madrid: CYAN Proyectos Editoriales S.A.

Moresco, B. L. et al. (2018): “A quiet disease with loud manifestations”. Semin Pediatr Neurol, 26: 88-91.

Morton, C. C. y Nance, W. E. (2006): “Newborn hearing screening. A silent revolution”. N Engl J Med, 354: 21512164.

Moteki, H. et al. (2018): "A rational approach to identifying newborns with hearing loss caused by congenital cytomegalovirus infection by dried blood spot screening”. Acta Otolaryngol, 138: 708-712.

Nance, W. E. et al. (2006): "Importance of congenital cytomegalovirus infections as a cause for pre-lingual hearing loss”. J. Clin. Virol, 35: 221-225. 
Nikolopoulos, T. P. (2015): "Neonatal hearing screening. What we have achieved and what needs to be improved". Int J Pediatr Otorhinolaryngol, 79: 635-637.

Nuñez-Ramos, R. et al. (2013): "Early diagnosis of congenital cytomegalovirus infection: lost opportunities". Enferm Infecc Microbiol Clin, 31: 93-96.

Park, A. H. et al. (2014): "A diagnostic paradigm including cytomegalovirus testing for idiopathic pediatric sensorineural hearing loss”. Laryngoscope, 124: 2624-2629.

Peng, Q. et al. (2016): "Concurrent genetic and standard screening for hearing impairment in 9317 southern chinese newborns". Genet Test Mol Biomarkers, 20: 603-608.

Pimperton, H. et al. (2017): "Language outcomes in deaf or hard of hearing teenagers who are spoken language users: effects of universal newborn hearing screening and early confirmation". Ear Hear, 38: 598-610.

Pimperton, H. et al. (2016): "The impact of universal newborn hearing screening on long-term literacy outcomes: a prospective cohort study”. Arch Dis Child, 101: 9-15.

Pryor, S. P. et al. (2005): "SLC26A4/PDS genotype phenotype correlation in hearing loss with enlargement of the vestibular aqueduct (EVA): evidence that Pendred syndrome and non-syndromic EVA are distinct clinical and genetic entities". J Med Genet, 42: 159-165.

Ravi, R. et al. (2016): "Follow-up in newborn hearing screening. A systematic review". Intl J Pediatr Otorhinolaryngol, 90: 29-36.

Rawlinson, W. D. et al. (2018): "Neonates with congenital cytomegalovirus and hearing loss identified via the universal newborn hearing screening program". J ClinVirol, 102: 110-115.

Rawlinson, W. D. et al. (2017): "Congenital cytomegalovirus infection in pregnancy and the neonate: Consensus recommendations for prevention, diagnosis, and therapy". Lancet Infect Dis, 17: e177-188.

Ross, S. A. et al. (2017): "Newborn dried blood spot polymerase chain reaction to identify infants with congenital cytomegalovirus-associated sensorineural hearing loss". J Pediatr, 184: 57-61.

Ross, S. A. et al. (2015): "National Institute on Deafness and Other Communication Disorders CHIMES Study. Urine collection method for the diagnosis of congenital cytomegalovirus infection". Pediatr Infect Dis, 34: 903-905.

Schimmenti, L. A. et al. (2011): "Evaluation of newborn screening bloodspot-based genetic testing as second tier screen for bedside newborn hearing screening". Genet Med, 13: 1006-1010.

Stehel, E. K. et al. (2008): "Newborn hearing screening and detection of congenital cytomegalovirus infection". Pediatrics, 121: 970-975.

Smiechura, M. et al. (2014): "Congenital and acquired cytomegalovirus infection and hearing evaluation in children". Otolaryngol Pol, 68: 303-307.

Sun, et al. (2015): "Combined hearing and deafness gene mutation screening of 11,046 Chinese newborns". Zhonghua Yi Xue Yi ChuanXueZaZhi, 32 (6): 766-770.

Teek, R. et al. (2013): "Hearing impairment in Estonia: an algorithm to investigate genetic causes in pediatric patients". Adv Med Sci, 58: 419-428.

Toumpas, C. J. et al. (2015): "Congenital cytomegalovirus infection is a significant cause of moderate to profound sensorineural hearing loss in Queensland children”. J Paediatr Child Health, 51: 541-544. 
Trinidad, G. et al. (2010): "Recomendaciones de la Comisión para la Detección Precoz de la Hipoacusia (CODEPEH) para 2010". Acta Otorrinolaringol Esp, 61: 69-77.

Vancor, E. et al. (2019): "Results of a targeted screening program for congenital cytomegalovirus infection in infants who fail newborn hearing screening". J Pediatric Infect Dis Soc, 8 (1): 55-59.

Vives-Oñós, I. et al. (2018): "Is Polymerase Chain Reaction in Neonatal Dried Blood Spots Reliable for the Diagnosis of Congenital Cytomegalovirus Infection?". Pediatr Infect Dis J, 38 (5): 520-524.

Wake, M. et al. (2016): "Population Outcomes of Three Approaches to Detection of Congenital Hearing Loss". Pediatrics, 137: 1-10.

Wang, Q. J. et al. (2011): "Newborn hearing concurrent gene screening can imporve care for hearing loss: a study on 14.913 chinese newborns". Int J Pediatr Otorhinolaryngol, 75: 535-542.

Watkin, P. y Baldwin, M. (2012): "The longitudinal follow up of a universal neonatal hearing screen: the implications for confirming deafness in childhood”. Int J Audiol, 51: 519-528.

Watkin, P. y Baldwin, M. (2011): "Identifying deafness in early childhood: requirements after the newborn hearing screening". Arc Dis Child, 96: 62-66.

Williams, E. J. et al. (2015): "First estimates of the potential cost and cost saving of protecting childhood hearing from damage caused by congenital CMV infection". Arch Dis Child Fetal Neonatal Ed, 100 (6): F501-506.

Williams, E. J. et al. (2014): "Feasibility and acceptability of targeted screening for congenital CMV-related hearing loss". Arch Dis Child Fetal Neonatal Ed, 99 (3): F230-236.

Wood, S. A. et al. (2015): "Performance and characteristics of the Newborn Hearing Screening Programme in England: The first seven years". Int J Audiol, 54: 353-358.

Wu, C. C. et al. (2017): "Newborn genetic screening for hearing impairment: a population-based longitudinal study". Genet Med, 19 (1): 6-12.

Yamaguchi, A. et al. (2017): "Screening for seemingly healthy newborns with congenital cytomegalovirus infection by quantitative real-time polymerase chain reaction using newborn urine: an observational study". BMJ Open, 7: e013810.

Young, N. M. et al. (2011): "Limitations of universal newborn hearing screening in early identification of pediatric cochlear implant candidates”. Arch Otolaryngol Head Neck Surg, 137: 230-234.

Zou, B. et al. (2015): “The application of genome editing in studying hearing loss”. Hear Res, 327: 102-108.

Zubicaray, J. et al. (2014): "Sistemática del cribado de la audición en el niño". En Manrique, M. y Marco, J. (coords.): Audiología, Ponencia Oficial de la Sociedad Española de Otorrinolaringología y Patología CérvicoFacial. Madrid: CYAN Proyectos Editoriales S.A. 